\title{
Conjunctival oxygen monitoring during cardiopulmonary resuscitation
}

Sir

I read with interest the paper by J. Heyworth in the June 1989 edition of Archives of Emergency Medicine, 'Conjunctival oxygen monitoring during cardiopulmonary resuscitation'.

I have recently studied seven patients undergoing cardiopulmonary resuscitation (CPR) using a pulse oximeter. There is one previous report in the literature of a pulse oximeter being used during CPR, in a child who arrested during an anaesthetic as a \& result of an overdose of a potassium salt of penicillin. The pulse oximeter showed $\vec{\circ}$ saturations above $90 \%$ during the $7 \mathrm{~min}$ of CPR and the child made an uneventful recovery. The pulse oximeter works by sensing the differential light absorption of oxy and reduced haemoglobin and hence calculating the percentage saturation. A microprocessor in the instrument substracts the 'background' absorption from that due to the pulsatile flow and hence derives the arterial saturation. The result of this is that the instrument will only function if there is an adequate flow for it. Pulse oximeters will give no reading if there is vascular stasis due for instance to extreme vasoconstriction or death.

In the seven patients studied using both ear and finger sensors we have found very similar results to J. Heyworth. There was one survivor in this series and her saturation stayed consistently above $91 \%$ although she flipped into and out of cardiac rhythms with an output during the period of resuscitation. The remaining six patients showed an initial rise in saturation as CPR was commenced but this had fallen to below $80 \%$ in a patients after $10 \mathrm{~min}$ of CPR.

These results, although only a small series, appear to correlate with those of Heyworth in that the arterial oxygenation did not seem to be sustained during closed CPR in those patients who died. However, we all have experience of patients who have undergone prolonged closed CPR and survived neurologically intact. Presumably these patients must have sustained reasonable cerebral oxygenation during the period of CPR.

\section{ACKNOWLEDGEMENTS}

I would like to thank Critikon for the loan of the Oxyshuttle pulse oximeter and Physiocontrol for the loan of the Lifestat 1600 pulse oximeter.

\section{R. JAMES}

Accident and Emergency Department,

Royal Preston Hospital,

Preston, Lancashire, England

\section{REFERENCE}

Naring V. P. S. (1986) Utility of the pulse oximeter during cardiopulmonary resuscitation. Anesthesiology 65, 239-40. 